\title{
Split operator finite element method for modelling pulmonary gas exchange
}

\author{
G. J. Bignell ${ }^{1} \quad$ P. R. Johnston ${ }^{2}$
}

(Received 31 August 2006; revised 26 July 2007)

\begin{abstract}
A pulmonary gas exchange model which contains six species of interest in three different regions is considered. The model leads to a system of coupled non-linear parabolic partial differential equations and is solved using a transport-reaction split operator scheme. A Galerkin weighted residual finite element method solves the transport algorithm while a simple forward time step is used for the reaction algorithm. Using different time scales for the transport and reaction algorithms, we obtain a reasonable approximation of gas exchange.
\end{abstract}

\section{Contents}

See http://anziamj.austms.org.au/ojs/index.php/ANZIAMJ/article/view/125 for this article, (c) Austral. Mathematical Soc. 2007. Published August 9, 2007. ISSN 1446-8735 


\section{The model}

2.1 Governing equations . . . . . . . . . . . . . C368

2.2 Reaction equations . . . . . . . . . . . . . . . . C369

2.3 Boundary and interface conditions . . . . . . . . . . C370

3 Developing a model solution

3.1 Split operator scheme . . . . . . . . . . . . . . C371

3.1 .1 Transport PDE . . . . . . . . . . . . . C372

3.1 .2 Reaction ODE . . . . . . . . . . . . C373

3.2 Boundary and interface conditions . . . . . . . . . . C374

3.2.1 The Gibbs-Donnan condition . . . . . . . . . C374

4 The model solution

C375

5 Conclusion

C376

A Model parameters

C376

References

C378

\section{Introduction}

Pulmonary gas exchange is essential to life. It involves the transfer of respiratory gases (oxygen and carbon dioxide) between the red blood cells and alveoli (air sacs in the lungs) via the respiratory membrane and blood plasma. Exchange occurs by three processes: diffusion, convection and chemical reaction. These processes occur on the microscopic scale and cannot be directly measured, thus mathematical models are required to investigate what is occurring within the pulmonary capillary and how quickly equilibrium is achieved.

The model considered in this article consists of twelve coupled non-linear 
parabolic partial differential equations (PDEs) which apply to six different species in three different regions. A transport-reaction split operator scheme is used to deal with the non-linearity of the reaction terms. The split gives a transport PDE, which includes the diffusion and convection terms, and a reaction ordinary differential equation (ODE), which contains the reaction terms. The transport PDE is solved using a Galerkin weighted residual finite element method with a Crank-Nicolson iteration scheme. The reaction ODE uses a simple forward time step.

A program has been written in $\mathrm{C}$ to solve the transport-reaction split operator model. The program handles multiple coupled equations and regions as well as changes in boundary conditions. We found that the transport and reaction algorithms run on different time scales $\left(\Delta t=10^{-4} \mathrm{~s}\right.$ and $10^{-6} \mathrm{~s}$ respectively). By performing 100 reaction iterations for every transport iteration a reasonable solution is obtained and computing time minimised.

The model geometry and equations presented in this article are based upon a paper by Whiteley, Gavaghan and Hahn [1]. The aim was to reproduce the results given by Whiteley et al. [1] to test the accuracy and stability of the method and program. A number of omissions and inaccuracies given by Whiteley et al. [1] have also been addressed. Examples of these are the incorrect value of a constant $\left(K_{c}\right)$ and using a constant value instead of the given reaction equation $\left(R_{2}\right)$ [2]. Also, all convection terms were neglected by Whiteley et al. [1] whereas in this article the full stated equations were solved.

\section{The model}

To mathematically model the complex anatomical structures present in the lungs, various simplifications and assumptions are needed. To obtain the required two dimensional geometry a section is taken through the length of the pulmonary capillary (CAP). The red blood cells in the capillary are assumed to be traveling in single file, evenly spaced and with constant velocity. The 
Alveolar Gas/Tissue

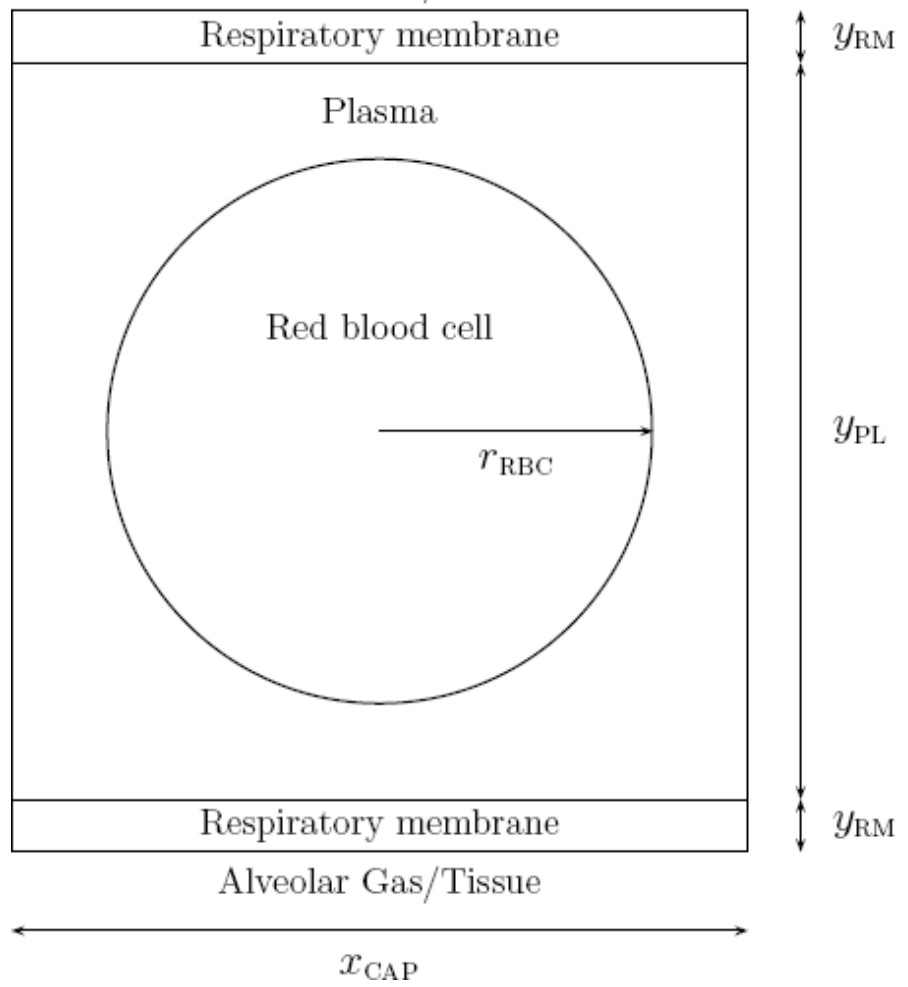

Figure 1: The two dimensional unit cell

model or unit cell, shown in Figure 1, consists of one red blood cell (RBC) surrounded by a region of plasma (PL), bound on two sides by a respiratory membrane (RM). As the RBC moves through the pulmonary circulation, the region adjacent to the respiratory membrane represents alveolar (ALV) gas then tissue. We assume that flux is only possible through the respiratory membranes and only while alveolar gas is present (when the RBC is within the pulmonary capillary). The reference frame is fixed in the RBC to account for the cell's movement through the pulmonary circulation.

There are three different reactions involved in pulmonary gas exchange. 
The oxyhaemoglobin reaction is the binding of oxygen $\left(\mathrm{O}_{2}\right)$ and haemoglobin $(\mathrm{Hb})$ to create oxyhaemoglobin $\left(\mathrm{HbO}_{2}\right)$. The carbaminohaemoglobin reaction is the unloading of carbon dioxide $\left(\mathrm{CO}_{2}\right)$ from carbaminohaemoglobin $\left(\mathrm{HbCO}_{2}\right)$. Both of these reactions occur in the RBC. The other reaction is the bicarbonate reaction which occurs in both the plasma and RBC. This is the combining of $\mathrm{CO}_{2}$ and water $\left(\mathrm{H}_{2} \mathrm{O}\right)$ to create bicarbonate $\left(\mathrm{HCO}_{3}^{-}\right)$and hydrogen $\left(\mathrm{H}^{+}\right)$ions. To get an accurate picture of what is occurring within the pulmonary capillary, six different species need to be monitored. These species are represented in the equations as

$$
\begin{array}{ll}
A=P_{\mathrm{O}_{2}} & \text { partial pressure of oxygen, } \\
B=P_{\mathrm{CO}_{2}} & \text { partial pressure of carbon dioxide, } \\
C=\left[\mathrm{H}^{+}\right] & \text {hydrogen ion concentration, } \\
E=\left[\mathrm{HCO}_{3}^{-}\right] & \text {bicarbonate ion concentration, } \\
F=S_{\mathrm{HbO}_{2}} & \text { oxyhaemoglobin saturation, } \\
G=S_{\mathrm{HbCO}_{2}} & \text { carbaminohaemoglobin saturation. }
\end{array}
$$

Appendix A lists typical initial and final values for each species, along with definitions and values for all other variables and coefficients.

\subsection{Governing equations}

Oxygen and carbon dioxide $(A$ and $B)$ are present in all areas of the model. Hydrogen and bicarbonate ions $(C$ and $E)$ are present where the reactions occur, namely the plasma and RBC. Oxyhaemoglobin and carbaminohaemoglobin $(F$ and $G)$ are found only within the RBC. Diffusion occurs within all regions of the model and, as the frame is fixed in the RBC, convection occurs in the respiratory membrane and plasma. This gives a total of twelve governing equations, which consist of two equations in the respiratory membrane,

$$
\frac{\partial A}{\partial t}=D_{A} \nabla^{2} A-\mathbf{v} \cdot \nabla A, \quad \frac{\partial B}{\partial t}=D_{B} \nabla^{2} B-\mathbf{v} \cdot \nabla B,
$$


four equations in the plasma,

$$
\begin{aligned}
& \frac{\partial A}{\partial t}=D_{A} \nabla^{2} A-\mathbf{v} \cdot \nabla A, \quad \frac{\partial B}{\partial t}=D_{B} \nabla^{2} B-\mathbf{v} \cdot \nabla B+\frac{R_{1}}{\alpha_{B}} \\
& \frac{\partial C}{\partial t}=D_{C} \nabla^{2} C-\mathbf{v} \cdot \nabla C-\frac{2.303 C}{\beta_{\mathrm{PL}}} R_{1}, \quad \frac{\partial E}{\partial t}=D_{E} \nabla^{2} E-\mathbf{v} \cdot \nabla E-R_{1},
\end{aligned}
$$

and six equations in the RBC,

$$
\begin{aligned}
& \frac{\partial A}{\partial t}=D_{A} \nabla^{2} A-\frac{R_{2}}{\alpha_{A}}, \quad \frac{\partial B}{\partial t}=D_{B} \nabla^{2} B+\frac{\gamma R_{1}-R_{3}}{\alpha_{B}} \\
& \frac{\partial C}{\partial t}=D_{C} \nabla^{2} C+\frac{2.303 C}{\beta_{\mathrm{RBC}}}\left(-\gamma R_{1}-0.6 R_{2}+1.5 R_{3}\right) \\
& \frac{\partial E}{\partial t}=D_{E} \nabla^{2} E-\gamma R_{1}, \quad \frac{\partial F}{\partial t}=D_{H} \nabla^{2} F+\frac{R_{2}}{c_{H}}, \quad \frac{\partial G}{\partial t}=D_{H} \nabla^{2} G+\frac{R_{3}}{c_{H}} .
\end{aligned}
$$

\section{$2.2 \quad$ Reaction equations}

In the governing equations, $R_{1}, R_{2}$ and $R_{3}$ are the bicarbonate, oxyhaemoglobin and carbaminohaemoglobin reaction rates respectively:

$$
\begin{aligned}
& R_{1}=-k_{u} \alpha_{B} B+\frac{k_{v}}{K} C E, \\
& R_{2}=k c_{H} \frac{f(A, B, C)-F}{1-f(A, B, C)}, \\
& R_{3}=c_{H} k_{a}\left(\alpha_{B} B v(C, F)+\frac{C}{K_{C}}\right)(g(B, C, F)-G) .
\end{aligned}
$$

The rates of $R_{2}$ and $R_{3}$ are affected by the $\mathrm{HbO}_{2}$ and $\mathrm{HbCO}_{2}$ saturations $\left(F\right.$ and $G$ ). The presence of bound $\mathrm{O}_{2}$ promotes the unloading of $\mathrm{CO}_{2}$, while at the same time unloaded $\mathrm{CO}_{2}$ increases haemoglobin's ability to bind $\mathrm{O}_{2}$ [3]. The equilibrium values of $F$ and $G$, which influence reaction rates, 
are calculated using

$$
\begin{aligned}
& f(P(A, B, C))= \begin{cases}\frac{a_{1} P+a_{2} P^{2}+a_{3} P^{3}+P^{4}}{a_{4}+a_{5} P+a_{6} P^{2}+a_{7} P^{3}+P^{4}}, & P \geq 12 \mathrm{mmHg}, \\
0.003683 P+0.000584 P^{2} & P<12 \mathrm{mmHg},\end{cases} \\
& g(B, C, F)=\frac{\alpha_{B} B v(C, F)}{\alpha_{B} B v(C, F)+C / K_{C}} .
\end{aligned}
$$

The other reaction equation terms are

$$
\begin{aligned}
& v(C, F)=\frac{K_{z o} F}{K_{z o}+C}+\frac{K_{z r}(1-F)}{K_{z r}+C}, \\
& P(A, B, C)=A \cdot 10^{0.40(\mathrm{pH}-7.4)+0.06 \log _{10}(40 / B),}
\end{aligned}
$$

where $\mathrm{pH}=-\log _{10}(r C)$. In the published results of Whiteley et al. [1], $R_{2}$ was set to equal $k c_{H}$ [2]. This means that the fraction in the reaction rate equation, which actually limits $\mathrm{O}_{2}$ and $\mathrm{Hb}$ binding, is no longer effective. Using the constant value instead of the reaction rate means the value of $F$ can potentially reach or exceed 1 . This gives a $\mathrm{HbO}_{2}$ saturation greater than $100 \%$ which is physiologically impossible.

Another factor that must be taken into account is the Gibbs-Donnan condition [4], defined as

$$
r=\left(0.058 \mathrm{pH}_{\mathrm{PL}}-0.437\right) F-0.529 \mathrm{pH}_{\mathrm{PL}}+4.6, \quad \mathrm{pH}_{\mathrm{PL}}=-\log _{10} C_{\mathrm{PL}},
$$

with $C_{\mathrm{PL}}$ given in Molar. This condition describes an electrical and osmotic equilibrium that exists between the plasma and RBC, caused by a shift of charged ions between the two regions. The ion shift occurs because $\mathrm{HCO}_{3}^{-}$ is predominately made in the RBCs but transported in the plasma.

\subsection{Boundary and interface conditions}

All species obey Neumann boundary conditions (zero flux) at the boundaries of the regions in which they are present. This is along the unit cell and 
ALV-RM boundaries for $A$ and $B$, along the unit cell boundaries and RMPL interfaces for $C$ and $E$, and the PL-RBC interface for $F$ and $G$. The only exception to the Neumann condition is while the RBC is within the pulmonary capillary $A$ and $B$ obey Dirichlet boundary conditions such that $A=A_{\mathrm{ALV}}$ and $B=B_{\mathrm{ALV}}$ along the ALV-RM boundary.

There are also interface conditions which must be obeyed along the internal interfaces. $A$ and $B$ obey the continuity conditions,

$$
\begin{aligned}
& A_{\mathrm{RM}}=A_{\mathrm{PL}}, \quad \frac{\partial A_{\mathrm{RM}}}{\partial y}=\frac{\partial A_{\mathrm{PL}}}{\partial y}, \quad B_{\mathrm{RM}}=B_{\mathrm{PL}}, \quad \frac{\partial B_{\mathrm{RM}}}{\partial y}=\frac{\partial B_{\mathrm{PL}}}{\partial y}, \\
& A_{\mathrm{PL}}=A_{\mathrm{RBC}}, \quad \frac{\partial A_{\mathrm{PL}}}{\partial n}=\frac{\partial A_{\mathrm{RBC}}}{\partial n}, \quad B_{\mathrm{PL}}=B_{\mathrm{RBC}}, \quad \frac{\partial B_{\mathrm{PL}}}{\partial n}=\frac{\partial B_{\mathrm{RBC}}}{\partial n}
\end{aligned}
$$

along the RM-PL and PL-RBC interfaces. At the PL-RBC interface, $C$ and $E$ obey the discontinuity (or Gibbs-Donnan) condition,

$$
r=\frac{C_{\mathrm{PL}}}{C_{\mathrm{RBC}}}=\frac{E_{\mathrm{RBC}}}{E_{\mathrm{PL}}},
$$

where $r$ was defined by (11). For more specific details on boundary and interface coordinates and conditions, see the article by Whiteley et al. [1].

\section{Developing a model solution}

\subsection{Split operator scheme}

The equations used in the model are non-linear coupled PDEs of the form

$$
\frac{\partial u}{\partial t}=D \nabla^{2} u-\mathbf{v} \cdot \nabla u+R\left(u, u_{1}, u_{2}, \ldots\right) .
$$

The first and second terms on the right hand side of (15) represent diffusion and convection respectively. These are linear and non-coupled. However, 
the third reaction term is non-linear and coupled. Solving the full non-linear coupled system is complex and time consuming. One way to simplify the solution is to apply a split operation scheme [5]. When applying this scheme the terms on the right are split to give one or more equations that equal the left hand term. This allows different terms to be solved using different methods, with the split being done in such a way as to simplify the solution algorithm and reduce computing time. In this case, the simplest way to apply a split operator scheme is to split the linear/non-coupled and nonlinear/coupled terms. The diffusion and convection terms in (15) are split from the reaction term to give a transport PDE and a reaction ODE:

$$
\frac{\partial u}{\partial t}=D \nabla^{2} u-\mathbf{v} \cdot \nabla u \quad \text { and } \quad \frac{d u}{d t}=R\left(u, u_{1}, u_{2}, \ldots\right) .
$$

Both of these equations are solved sequentially within each time step, with each equation solved over an entire time step. This apparent discrepancy is dealt with by solving the first equation using $u^{t}$ values to give $u^{t *}$ or intermediate values. These $u^{t *}$ values are then used to solve the second equation giving the required $u^{t+1}$ values. In order to reduce error, the PDE and ODE are alternately solved first within each time step [5].

\subsubsection{Transport PDE}

The transport PDE is solved using a Galerkin weighted residual finite element method (FEM) [6]. The model is discretised into triangular elements with linear basis functions, $\phi_{j}$, such that

$$
u=\sum_{j=1}^{n} \phi_{j} u_{j}+\sum_{j=n+1}^{m} \phi_{j} u_{j},
$$

where the $u_{j}$ values at nodes 1 to $n$ are unknown, and $n+1$ to $m$ are known or Dirichlet nodes. The transport PDE is multiplied by a weighting function, $\phi_{i}$, 
and integrated over a region. Using the Green-Gauss Theorem and after some algebra the equation

$$
\begin{aligned}
& \sum_{j=1}^{n}\left(\frac{D}{4 A}\left(b_{i} b_{j}+c_{i} c_{j}\right)+\frac{1}{6}\left(v_{x} b_{j}+v_{y} c_{j}\right)\right) u_{j}+\sum_{j=1}^{n}\left(\frac{A}{12}+\delta_{i j} \frac{A}{12}\right) \frac{d u_{j}}{d t} \\
& =\sum_{j=n+1}^{m}\left(-\frac{D}{4 A}\left(b_{i} b_{j}+c_{i} c_{j}\right)-\frac{1}{6}\left(v_{x} b_{j}+v_{y} c_{j}\right)\right) u_{j}
\end{aligned}
$$

is obtained, where $A$ is the element area, $b_{i}, b_{j}, c_{i}$ and $c_{j}$ are element parameters, and $v_{x}$ and $v_{y}$ are velocity components. Equation (18) can be rewritten as the matrix equation $\mathbf{A u}+\mathbf{B} \dot{\mathbf{u}}=\mathbf{C}$. Using a Crank-Nicolson Scheme where $\dot{\mathbf{u}}=\left(\mathbf{u}^{t+1}-\mathbf{u}^{t}\right) / \Delta t$ and $\mathbf{u}=\left(\mathbf{u}^{t+1}+\mathbf{u}^{t}\right) / 2$, rearrange the matrix equation to

$$
\left(\frac{\mathbf{A}}{2}+\frac{\mathbf{B}}{\Delta t}\right) \mathbf{u}^{t+1}=\left(-\frac{\mathbf{A}}{2}+\frac{\mathbf{B}}{\Delta t}\right) \mathbf{u}^{t}+\mathbf{C},
$$

which upon simplification gives $\mathbf{R} \mathbf{u}^{t+1}=\mathbf{S u}^{t}+\mathbf{C}=\mathbf{T}$. In this matrix equation, $\mathbf{u}^{t+1}$ is a vector of unknown values which is solved using an LU decomposition of $\mathbf{R}$ and back substitution [7].

\subsubsection{Reaction ODE}

The reaction ODE is solved using a simple forward time step:

$$
\frac{u^{t+1}-u^{t}}{\Delta t}=R\left(u^{t}, u_{1}^{t}, u_{2}^{t}, \ldots\right) .
$$

The term on the right is a function of the three reaction rates, $R_{1}, R_{2}$ and $R_{3}$, each multiplied by an appropriate constant. Letting the term $R\left(u^{t}, u_{1}^{t}, u_{2}^{t}, \ldots\right)$ be represented by $c_{1} R_{1}^{t}+c_{2} R_{2}^{t}+c_{3} R_{3}^{t}$, equation (20) rearranges to

$$
u^{t+1}=u^{t}+\Delta t\left(c_{1} R_{1}^{t}+c_{2} R_{2}^{t}+c_{3} R_{3}^{t}\right) .
$$


The reaction rates are functions of more than one species, so there is only one value for each of $R_{1}, R_{2}$ and $R_{3}$ calculated at each node. Using the same nodes from the FEM discretisation, reaction rate values are calculated node by node and then equation (21) solved for each species.

\subsection{Boundary and interface conditions}

Two types of boundary conditions are used in the model, Neumann and Dirichlet. All boundaries are of the Neumann type except at the ALV-RM boundary which changes from Dirichlet to Neumann as the RBC passes out of the pulmonary capillary. When solving the Transport PDE, the Neumann condition is implicit in the solution. However, the Dirichlet boundary conditions are explicitly included on the right side of (18) as the known $u_{j}$ values. When solving the Reaction ODE, boundary conditions are ignored.

There are also two types of interface condition within the model: continuity and discontinuity. The continuity condition, or continuous flux and concentration across interfaces, is implicit in the FEM equations and not relevant for the reaction equation. However, the discontinuity or GibbsDonnan condition must be specifically included in both the reaction and transport equations.

\subsubsection{The Gibbs-Donnan condition}

The Gibbs-Donnan condition, (14), must be satisfied for $C$ and $E$ on the PLRBC interface, where $r$ and $\mathrm{pH}_{\mathrm{PL}}$ are defined by (11). From these equations see that $r$ is a function of $C$ in the plasma and $F$ in the RBC. To ensure that the Gibbs-Donnan condition is satisfied the nodes on the PL-RBC interface are considered to be part of the plasma for $C$ and $E$ and part of the RBC for $A, B, F$ and $G$. A value for $r$ is now easily calculated at each of these 
nodes. To apply the Gibbs-Donnan condition, equation (14) rearranges to

$$
C_{\mathrm{RBC}}=\frac{C_{\mathrm{PL}}}{r} \quad \text { and } \quad E_{\mathrm{RBC}}=r E_{\mathrm{PL}} .
$$

When applying the FEM, elements in the plasma region that include PLRBC interface nodes ignore the Gibbs-Donnan condition as all nodes are considered to be in the plasma. However, RBC elements that include interface nodes must use the definitions for $C_{\mathrm{RBC}}$ and $E_{\mathrm{RBC}}$ given in (22) to calculate the correct values at each interface node. When solving the reaction ODE the Gibbs-Donnan condition is ignored at all nodes except the PL-RBC interface nodes. At these nodes (22) is applied to give all species their correct $\mathrm{RBC}$ value.

\section{The model solution}

Short simulations using various configurations were run to get a feel for the stability and accuracy of the program and method, and the time it would take to run larger simulations. We found that the solution of the transport PDE was stable when $\Delta t \leq 10^{-4} \mathrm{~s}$. The solution of the full transport-reaction algorithm appears reasonable only when $\Delta t \leq 10^{-6} \mathrm{~s}$. Further examination of this difference in time step size showed that the concentration change caused by reactions was much greater than that caused by transport. To deal with this difference the two algorithms were run on different time scales, $\Delta t_{\text {transport }}=10^{-4} \mathrm{~s}$ and $\Delta t_{\text {reaction }}=10^{-6} \mathrm{~s}$. For every transport iteration the program performed 100 reaction iterations, cutting down the total number of iterations needed and greatly reducing computing time.

Testing showed that the solution proved very sensitive to step size. If a time step was too large, the solution values would oscillate between very large positive and negative numbers, giving totally unreasonable results. We also estimated that a full simulation, using a course grid, would take over two 
months to run. These two facts show that the solution method is impractical, so it is considered necessary to find different solution techniques that allow for larger time steps and hence, shorter run times. However, we found that the transport-reaction split operator scheme provided a good basis upon which to build.

\section{Conclusion}

A transport-reaction split operator scheme has been applied to solve a pulmonary gas exchange model, giving a transport PDE and a reaction ODE. The non-coupled linear transport PDE is solved using a Galerkin weighted residual FEM with a Crank-Nicolson iteration scheme. The coupled nonlinear reaction ODE is solved using a simple forward time step. The use of different time scales for the two algorithms gives a reasonably stable solution. However, many time steps are needed for a full solution which requires quite lengthy computing time. This large number of time steps to achieve reasonable stability may indicate that the forward time step solution for the reaction ODE is unstable. The next step in developing a model solver is to try applying a Crank-Nicolson iteration scheme to the reaction ODE. This will increase the work during each iteration, by giving a system of equations to be solved at each node, but it should reduce the total number of iterations needed.

\section{A Model parameters}

\section{Species Initial value Final value}

A

$A_{\mathrm{ALV}}$

$40 \mathrm{mmHg}$

$100 \mathrm{mmHg}$

$B$

$100 \mathrm{mmHg}$

$46 \mathrm{mmHg}$

$40 \mathrm{mmHg}$ 


$\begin{array}{lll}B_{\mathrm{ALV}} & 40 \mathrm{mmHg} & - \\ C_{\mathrm{PL}} & 4.23 \times 10^{-8} \mathrm{M} & 3.98 \times 10^{-8} \mathrm{M} \\ C_{\mathrm{RBC}} & 6.10 \times 10^{-8} \mathrm{M} & 5.70 \times 10^{-8} \mathrm{M} \\ E_{\mathrm{PL}} & 2.40 \times 10^{-2} \mathrm{M} & 2.26 \times 10^{-2} \mathrm{M} \\ E_{\mathrm{RBC}} & 1.70 \times 10^{-2} \mathrm{M} & 1.53 \times 10^{-2} \mathrm{M} \\ F & 0.72 & 0.97 \\ G & 0.11 & 0.06\end{array}$

Constant Value

$D_{A}$

$D_{B}$

$D_{C}$

$D_{E}$

$D_{H}$

$\mathbf{v}$

$\alpha_{A}$

$\alpha_{B}$

$\beta_{\mathrm{PL}}$

$\beta_{\mathrm{RBC}}$

$\gamma$

$c_{H}$

$k_{u}$

$k_{v}$

K

$k$

$k_{a}$

$K_{z o}$

$K_{z r}$

$K_{c}$

$t_{\mathrm{CAP}}$

$x_{\mathrm{CAP}}$

$y_{\mathrm{RM}}$

$0.75 \mathrm{~s}$
$2.40 \times 10^{-7} \mathrm{dm}^{2} \cdot \mathrm{s}^{-1}$

$1.76 \times 10^{-7} \mathrm{dm}^{2} . \mathrm{s}^{-1}$

$9.52 \times 10^{-7} \mathrm{dm}^{2} . \mathrm{s}^{-1}$

$1.25 \times 10^{-7} \mathrm{dm}^{2} \cdot \mathrm{s}^{-1}$

$1.40 \times 10^{-9} \mathrm{~s}^{-1}$

$(0.04,0.00) \mathrm{dm} . \mathrm{s}^{-1}$

$1.40 \times 10^{-6} \mathrm{M}^{-\mathrm{mmHg}^{-1}}$

$3.03 \times 10^{-5}{\mathrm{M} . \mathrm{mmHg}^{-1}}^{-1}$

$5.77 \times 10^{-3} \mathrm{M} \mathrm{H}^{+} / \mathrm{pH}$

$6.00 \times 10^{-2} \mathrm{M} \mathrm{H}^{+} / \mathrm{pH}$

$1.30 \times 10^{4}$

$2.00 \times 10^{-2} \mathrm{M}$

$1.20 \times 10^{-1} \mathrm{~s}^{-1}$

$8.90 \times 10^{1} \mathrm{~s}^{-1}$

$5.50 \times 10^{-4} \mathrm{M}$

$4.40 \times 10^{1} \mathrm{~s}^{-1}$

$5.00 \times 10^{3} \mathrm{M}^{-1} \cdot \mathrm{s}^{-1}$

$8.40 \times 10^{-9} \mathrm{M}$

$7.20 \times 10^{-8} \mathrm{M}$

$2.40 \times 10^{-5}$

$7.00 \times 10^{-5} \mathrm{dm}$

$0.50 \times 10^{-5} \mathrm{dm}$

\section{Definition}

$\mathrm{O}_{2}$ diffusion coefficient

$\mathrm{CO}_{2}$ diffusion coefficient

$\mathrm{H}^{+}$diffusion coefficient

$\mathrm{HCO}_{3}^{-}$diffusion coefficient

$\mathrm{Hb}$ diffusion coefficient

RBC velocity

$\mathrm{O}_{2}$ solubility

$\mathrm{CO}_{2}$ solubility

plasma buffer capacity

intracellular $\mathrm{Hb}$ capacity

carbonic anhydrase catalysis factor

$\mathrm{Hb}$ concentration

$\mathrm{HCO}_{3}^{-}$reaction forward rate

$\mathrm{HCO}_{3}^{-}$reaction backward rate

$\mathrm{HCO}_{3}$ dissociation equilibrium

$\mathrm{O}_{2}$ dissociation rate

$\mathrm{HbCO}_{2}$ reaction forward rate

$\mathrm{HbO}_{2}$ amino group ionisation

deoxy-Hb amino group ionisation $\mathrm{HbCO}_{2}$ ionisation

time RBC is within capillary

unit cell capillary length

respiratory membrane thickness 


$\begin{array}{lll}y_{\mathrm{PL}} & 7.00 \times 10^{-5} \mathrm{dm} & \text { capillary (plasma region) width } \\ r_{\mathrm{RBC}} & 2.60 \times 10^{-5} \mathrm{dm} & \text { RBC radius } \\ a_{1} & -8.5322289 \times 10^{3} & \\ a_{2} & 2.1214010 \times 10^{3} & \\ a_{3} & -6.7073989 \times 10 & \\ a_{4} & 9.3596087 \times 10^{5} & \\ a_{5} & -3.1346258 \times 10^{4} & \\ a_{6} & 2.3961674 \times 10^{3} & \\ a_{7} & -6.7104406 \times 10 & \end{array}$

\section{References}

[1] Whiteley, J. P., Gavaghan, D. J., Hahn, C. E. W. (2003). Mathematical modelling of pulmonary gas transport. Journal of Mathematical Biology, 47, 79-99. doi:10.1007/s00285-003-0196-8 C366, C370, C371

[2] Whiteley, J. P. (2005). Personal communication. C366, C370

[3] Des Jardins, T. (1998). Cardiopulmonary Anatomy and Physiology: Essentials For Respiratory Care (3rd ed.). New York: Delmar Publishers. C369

[4] Hill, E. P., Power, G. G., Longo, L. D. (1973). Mathematical simulation of pulmonary $\mathrm{O}_{2}$ and $\mathrm{CO}_{2}$ exchange. American Journal of Physiology, 224, 904-917. http:

//ajplegacy . physiology.org/cgi/content/citation/224/4/904 C370

[5] Simpson, M. J., Landman, K. A., Clement, T. P. (2005). Assessment of a non-traditional operator split algorithm for simulation of reactive transport. Mathematics and Computers in Simulation, 70, 44-60. doi:10.1016/j.matcom.2005.03.019 C372 
[6] Fletcher, C. A. J. (1984). Computational Galerkin Methods. New York: Springer-Verlag. C372

[7] Press, W. H., Teukolsky, S. A., Vetterling, W. T., Flannery, B. P. (1992). Numerical Recipes in C: The art of scientific computing (2nd ed.). Cambridge: Cambridge University Press. C373 


\section{Author addresses}

1. G. J. Bignell, School of Biomolecular and Physical Sciences, Griffith University, Nathan, Australia.

mailto:g.bignell@griffith.edu.au

2. P. R. Johnston, School of Biomolecular and Physical Sciences, Griffith University, Nathan, Australia. 